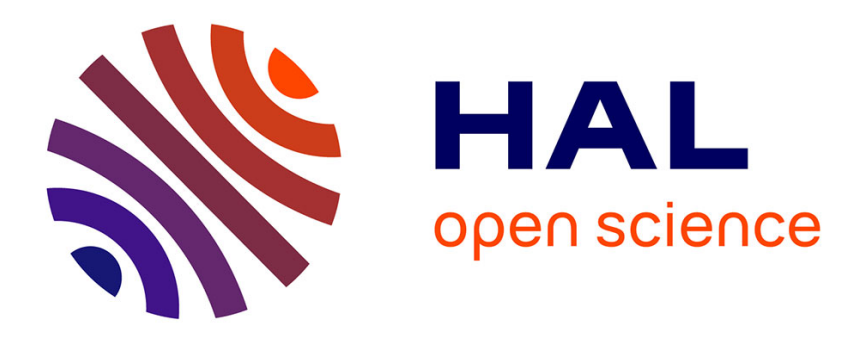

\title{
Regularization of Linear-Quadratic Control Problems with L 1 -Control Cost
}

Christopher Schneider, Walter Alt

\section{To cite this version:}

Christopher Schneider, Walter Alt. Regularization of Linear-Quadratic Control Problems with L 1 Control Cost. 26th Conference on System Modeling and Optimization (CSMO), Sep 2013, Klagenfurt, Austria. pp.296-305, 10.1007/978-3-662-45504-3_29 . hal-01286438

\section{HAL Id: hal-01286438 \\ https://hal.inria.fr/hal-01286438}

Submitted on 10 Mar 2016

HAL is a multi-disciplinary open access archive for the deposit and dissemination of scientific research documents, whether they are published or not. The documents may come from teaching and research institutions in France or abroad, or from public or private research centers.
L'archive ouverte pluridisciplinaire HAL, est destinée au dépôt et à la diffusion de documents scientifiques de niveau recherche, publiés ou non, émanant des établissements d'enseignement et de recherche français ou étrangers, des laboratoires publics ou privés.

\section{(c)(1)}

Distributed under a Creative Commons Attribution| 4.0 International License 


\title{
Regularization of linear-quadratic control problems with $L^{1}$-control cost
}

\author{
Christopher Schneider and Walter Alt \\ Fakultät für Mathematik und Informatik \\ Friedrich-Schiller-Universität \\ 07740 Jena, Germany \\ \{christopher.schneider, walter.alt\}@uni-jena.de
}

\begin{abstract}
We analyze $L^{2}$-regularization of a class of linear-quadratic optimal control problems with an additional $L^{1}$-control cost depending on a parameter $\beta$. To deal with this nonsmooth problem we use an augmentation approach known from linear programming in which the number of control variables is doubled. It is shown that if the optimal control for a given $\beta^{*} \geq 0$ is bang-zero-bang, the solutions are continuous functions of the parameter $\beta$ and the regularization parameter $\alpha$. Moreover we derive error estimates for Euler discretization.
\end{abstract}

Keywords: Optimal Control, Bang-Bang Control, $L^{1}$-Minimization, Nonsmooth Analysis, Regularization, Discretization.

\section{Introduction}

The regularization of optimal control problems by a $L^{2}$-term $\frac{\alpha}{2}\|u\|_{L^{2}}^{2}$ is often used in order to get a smoother optimal control. In this cases $\alpha$ can be viewed as a regularization parameter and one is interested in the question how the solutions depend on this parameter. For the special case that the control variable appears linearly in the control problem and the optimal control without regularization $(\alpha=0)$ has bang-bang structure this question has been investigated in Deckelnick/Hinze [1 for a class of elliptic control problems and in Alt/Seydenschwanz [2 for a general class of linear-quadratic control problems governed by ordinary differential equations.

Maurer/Vossen 3 investigate first order necessary and second order sufficient optimality conditions for a class of nonlinear control problems involving a $L^{1}$-term in the cost functional, where the parameter $\beta$ is kept fixed. They also propose some numerical algorithms for the solution of such problems. Sakawa [4] also considers a special numerical algorithm for a fixed parameter $\beta>0$. Stadler [ 5 and Casas et al. 617] investigate classes of elliptic control problems with a $L^{1}$-term in the cost functional, which is interpreted as a regularization term. They derive results on the dependence of the solutions on the parameter $\beta$ and error estimates for discretizations, but an additional $L^{2}$-regularization term with fixed parameter $\alpha$ is used in order to get smoother solutions. In Wachsmuth/Wachsmuth 8 the dependence of solutions of a class 
of elliptic control problems on the regularization parameter $\alpha$ is studied while the parameter $\beta$ is kept fix.

Results for the dependence of the solutions on the parameter $\beta$ and error estimates for discretizations for a general class of linear-quadratic control problems governed by ordinary differential equations have been recently derived in [9]. In the present paper, we investigate the regularization of such control problems and the dependence of solutions on the parameter $\beta$ and the regularization parameter $\alpha$ assuming that for a fixed parameter $\beta^{*}$ the corresponding optimal control is of bang-zero-bang type.

\section{Problem formulation}

With $X=X_{1} \times X_{2}, X_{1}=W_{\infty}^{1}\left(0, t_{f} ; \mathbb{R}^{n}\right), X_{2}=L^{\infty}\left(0, t_{f} ; \mathbb{R}^{m}\right)$, we consider the following family of $L^{2}$-regularized linear-quadratic control problems with $L^{1}$-control cost depending on the parameters $\alpha \geq 0$ and $\beta \geq 0$ :

$$
\begin{aligned}
& \min _{(x, u) \in X} f_{\alpha, \beta}(x, u) \\
& \begin{array}{ll}
\text { s.t. } \quad \dot{x}(t) & =A(t) x(t)+B(t) u(t) \quad \text { a.e. on }\left[0, t_{f}\right], \\
& x(0)=a,
\end{array} \\
& u(t) \in U \quad \text { a.e. on }\left[0, t_{f}\right],
\end{aligned}
$$

where $f_{\alpha, \beta}$ is a linear-quadratic cost functional with an additional nonsmooth $L^{1}$-term defined by

$$
\begin{aligned}
f_{\alpha, \beta}(x, u)= & \frac{1}{2} x\left(t_{f}\right)^{\top} Q x\left(t_{f}\right)+q^{\top} x\left(t_{f}\right) \\
& +\int_{0}^{t_{f}} \frac{1}{2} x(t)^{\top} W(t) x(t)+w(t)^{\top} x(t)+r(t)^{\top} u(t) \mathrm{d} t \\
& +\beta\|u\|_{L^{1}}+\frac{\alpha}{2}\|u\|_{L^{2}}^{2} .
\end{aligned}
$$

Here, $u(t) \in \mathbb{R}^{m}$ is the control, and $x(t) \in \mathbb{R}^{n}$ is the state of the system at time $t$, where $t \in\left[0, t_{f}\right]$. Further $Q \in \mathbb{R}^{n \times n}$ is a symmetric and positive semidefinite matrix, $q \in \mathbb{R}^{n}$, and the functions $W:\left[0, t_{f}\right] \rightarrow \mathbb{R}^{n \times n}, w:\left[0, t_{f}\right] \rightarrow \mathbb{R}^{n}$, $r:\left[0, t_{f}\right] \rightarrow \mathbb{R}^{m}, A:\left[0, t_{f}\right] \rightarrow \mathbb{R}^{n \times n}$, and $B:\left[0, t_{f}\right] \rightarrow \mathbb{R}^{n \times m}$ are Lipschitz continuous. The matrices $W(t)$ are assumed to be symmetric and positive semidefinite, and the set $U \in \mathbb{R}^{m}$ is defined by lower and upper bounds, i.e.

$$
U=\left\{u \in \mathbb{R}^{m} \mid b_{\ell} \leq u \leq b_{u}\right\}
$$

with $b_{\ell}, b_{u} \in \mathbb{R}^{m}, b_{\ell}<b_{u}$, where all inequalities are to be understood componentwise.

While the regularization term $\frac{\alpha}{2}\|u\|_{L^{2}}^{2}$ leads to a smooth optimal control for $\alpha>0$ the term $\beta\|u\|_{L^{1}}$ may be interpreted as both a regularization or some (nonsmooth) $L^{1}$-control cost. We are interested in the behavior of a solution $u^{\alpha, \beta}$ of Problem $\left(\sqrt{\mathrm{PQ}_{\alpha, \beta}}\right)$ depending on both parameters $\alpha$ and $\beta$. 


\section{Optimality conditions}

We denote by

$$
\mathcal{U}=\left\{u \in X_{2} \mid u(t) \in U \text { a.e. on }\left[0, t_{f}\right]\right\}
$$

the set of admissible controls, and by

$$
\mathcal{F}=\left\{(x, u) \in X \mid u \in \mathcal{U}, \dot{x}(t)=A(t) x(t)+B(t) u(t) \text { a.e. on }\left[0, t_{f}\right], x(0)=a\right\}
$$

the feasible set of $\left(\mathrm{PQ}_{\alpha, \beta}\right)$. Since $\mathcal{U}$ is nonempty, the feasible set $\mathcal{F}$ is nonempty, too. And since $\mathcal{U}$ is bounded, it follows that $\dot{x}$ is bounded for any feasible pair $(x, u) \in \mathcal{F}$, and therefore $\mathcal{F} \subset X$. Moreover, there is some constant $c$ such that $\|x\|_{1, \infty} \leq c\|u\|_{L^{\infty}}$ for any solution $x$ of the system equation, which implies that $\mathcal{F}$ is bounded.

A feasible pair $\left(x^{\alpha, \beta}, u^{\alpha, \beta}\right) \in \mathcal{F}$ is called a minimizer for Problem $\left(\mathrm{PQ}_{\alpha, \beta}\right)$ if $f_{\alpha, \beta}\left(x^{\alpha, \beta}, u^{\alpha, \beta}\right) \leq f_{\alpha, \beta}(x, u)$ for all $(x, u) \in \mathcal{F}$. Since the feasible set $\mathcal{F}$ is nonempty, closed, convex and bounded, and the cost functional is convex and continuous, a minimizer $\left(x^{\alpha, \beta}, u^{\alpha, \beta}\right) \in W_{2}^{1}\left(0, t_{f} ; \mathbb{R}^{n}\right) \times L^{2}\left(0, t_{f} ; \mathbb{R}^{m}\right)$ of $\left(\mathrm{PQ}_{\alpha, \beta}\right)$ exists (see [10, Chap. II, Prop. 1.2]), and since $\mathcal{U}$ is bounded we have $\left(x^{\alpha, \beta}, u^{\alpha, \beta}\right) \in X=W_{\infty}^{1}\left(0, t_{f} ; \mathbb{R}^{n}\right) \times L^{\infty}\left(0, t_{f} ; \mathbb{R}^{m}\right)$.

Let $\left(x^{\alpha, \beta}, u^{\alpha, \beta}\right) \in \mathcal{F}$ be a minimizer of $\left(\mathrm{PQ}_{\alpha, \beta}\right)$. Then there exist an element $\gamma^{\alpha, \beta} \in \partial\left\|u^{\alpha, \beta}\right\|_{L^{1}}$ of the subdifferential of $\left\|u^{\alpha, \beta}\right\|_{L^{1}}$ and a function $\lambda^{\alpha, \beta} \in$ $W_{\infty}^{1}\left(0, t_{f} ; \mathbb{R}^{n}\right)$ such that the adjoint equation

$$
\begin{aligned}
-\dot{\lambda}^{\alpha, \beta}(t) & =A(t)^{\top} \lambda^{\alpha, \beta}(t)+W(t) x^{\alpha, \beta}(t)+w(t) \quad \text { a.e. on }\left[0, t_{f}\right], \\
\lambda^{\alpha, \beta}\left(t_{f}\right) & =Q x^{\alpha, \beta}\left(t_{f}\right)+q,
\end{aligned}
$$

and the minimum principle

$$
\left[B(t)^{\top} \lambda^{\alpha, \beta}(t)+r(t)+\alpha u^{\alpha, \beta}(t)+\beta \gamma^{\alpha, \beta}(t)\right]^{\top}\left(u-u^{\alpha, \beta}(t)\right) \geq 0 \quad \forall u \in \mathcal{U}
$$

hold a.e. on $\left[0, t_{f}\right]$ (compare e.g. [11, Theorem 10.47] or [3, Sect. 2]).

Remark 1. Since $\left(\overline{\mathrm{PQ}_{\alpha, \beta}}\right)$ is a convex optimization problem for all $\alpha \geq 0$ and $\beta \geq 0$, a pair $\left(x^{\alpha, \beta}, u^{\alpha, \beta}\right) \in \mathcal{F}$ satisfying the minimum principle (2) and solving the adjoint equation (1) with some functions $\gamma^{\alpha, \beta}$ and $\lambda^{\alpha, \beta}$ is a solution of $\left(\mathrm{PQ}_{\alpha, \beta}\right)$ (compare [11, Propositon 4.12]).

Provided $\alpha=0$ we are able to evaluate the minimum principle (2) in more detail (compare [3] and [9]) and obtain

$$
u_{i}^{0, \beta}(t)= \begin{cases}b_{u, i}, & \text { if } \xi_{i}^{\beta}(t)<-\beta, \\ \text { undetermined } \left.\in] 0, b_{u, i}\right], & \text { if } \xi_{i}^{\beta}(t)=-\beta, \\ 0, & \text { if } \left.\xi_{i}^{\beta}(t) \in\right]-\beta, \beta[, \\ \text { undetermined } \in\left[b_{\ell, i}, 0[,\right. & \text { if } \xi_{i}^{\beta}(t)=\beta, \\ b_{\ell, i}, & \text { if } \xi_{i}^{\beta}(t)>\beta,\end{cases}
$$


where $\xi^{\beta}(t):=B(t)^{\top} \lambda^{0, \beta}(t)+r(t)$. If we assume that the set of switching times

$$
\mathfrak{M}_{i}^{\beta}=\left\{t \in\left[0, t_{f}\right] \mid \xi_{i}^{\beta}(t)=\beta \text { or } \xi_{i}^{\beta}(t)=-\beta\right\} .
$$

is finite, then by (3) the $i$-th component of the optimal control has a bang-zerobang structure.

\section{Problem transformation}

In common with [3] and [9] we formulate a transformed problem $\left(\mathrm{TQ}_{\alpha, \beta}\right)$ in order to study the dependence of the optimal control on the parameters $\alpha$ and $\beta$. This is a well known augmentation approach from linear programming wherewith we obtain a linear-quadratic control problem with smooth cost functional (see e.g. [12]).

Introducing new controls $v \in \tilde{X}_{2}:=L^{\infty}\left(0, t_{f} ; \mathbb{R}^{2 m}\right)$ and using the matrix

$$
M:=\left(\begin{array}{lllll}
1-1 & & & & \\
& 1-1 & & & \\
& & \ddots & \ddots & \\
& & & & 1-1
\end{array}\right) \in \mathbb{R}^{m \times 2 m}
$$

we have

$$
\begin{aligned}
& \min _{(x, v) \in X_{1} \times \tilde{X}_{2}} \bar{f}_{\alpha, \beta}(x, v) \\
& \text { s.t. } \quad \dot{x}(t)=A(t) x(t)+\mathcal{B}(t) v(t) \quad \text { a.e. on }\left[0, t_{f}\right] \text {, } \\
& x(0)=a \text {, } \\
& v(t) \in V \quad \text { a.e. on }\left[0, t_{f}\right] \text {, }
\end{aligned}
$$

where $\mathcal{B}(t):=B(t) M$. There are new box constraints for the controls,

$$
V:=\left\{v \in \mathbb{R}^{2 m} \mid v \geq 0, v_{2 i-1} \leq b_{u, i}, v_{2 i} \leq-b_{\ell, i}, i=1, \ldots, m\right\},
$$

and $f_{\alpha, \beta}$ is a linear-quadratic cost functional:

$$
\begin{aligned}
\bar{f}_{\alpha, \beta}(x, v)= & \frac{1}{2} x\left(t_{f}\right)^{\top} Q x\left(t_{f}\right)+q^{\top} x\left(t_{f}\right) \\
& +\int_{0}^{t_{f}} \frac{1}{2} x(t)^{\top} W(t) x(t)+w(t)^{\top} x(t)+r(t)^{\top} M v(t) \mathrm{d} t \\
& +\beta\|M v\|_{L^{1}}+\frac{\alpha}{2}\|M v\|_{L^{2}}^{2} .
\end{aligned}
$$

With the same argumentation as above for Problem $\left(\overrightarrow{\mathrm{PQ}_{\alpha, \beta}}\right)$ we are able to show that a minimizer of Problem $\left(\mathrm{TQ}_{\alpha, \beta}\right)$ exists. We denote the set of admissible controls by

$$
\mathcal{V}=\left\{v \in \tilde{X}_{2} \mid v(t) \in V \text { a.e. on }\left[0, t_{f}\right]\right\}
$$


and the feasible set of Problem $\left(\mathrm{TQ}_{\alpha, \beta}\right)$ by $\mathcal{T} \subset X_{1} \times \tilde{X}_{2}$, where

$$
\mathcal{T}=\left\{(x, v) \mid v \in \mathcal{V}, \dot{x}(t)=A(t) x(t)+\mathcal{B}(t) v(t) \text { a.e. on }\left[0, t_{f}\right], x(0)=a\right\} .
$$

Although Problem $\left(\mathrm{TQ}_{\alpha, \beta}\right)$ admits controls with components $v_{2 i-1}, v_{2 i}$ being positive simultaneously, such controls cannot be optimal (see [3, Sect. 4], 9, Sect. 3], [12, p. 42 et seq.]). Therefore, all optimal controls satisfy

$$
v_{2 i-1}^{\alpha, \beta}(t)=\max \left\{0, u_{i}^{\alpha, \beta}(t)\right\}, \quad v_{2 i}^{\alpha, \beta}(t)=\max \left\{0,-u_{i}^{\alpha, \beta}(t)\right\} .
$$

The optimality conditions also prove this result. By (5) and $v(t) \geq 0$ we now are able to simplify

$$
\|M v\|_{L^{1}}=\|v\|_{L^{1}}=\int_{0}^{t_{f}} \sum_{i=1}^{2 m} v_{i}(t) \mathrm{d} t \quad \text { and } \quad\|M v\|_{L^{2}}^{2}=\|v\|_{L^{2}}^{2},
$$

which nicely shows, that a $L^{1}$ - or $L^{2}$-regularization of the original problem implies the same regularization of the transformed problem. We finally introduce the minimum principle of Problem $\left(\mathrm{TQ}_{\alpha, \beta}\right)$

$$
\left[\sigma^{\alpha, \beta}\right]^{\top}\left(v-v^{\alpha, \beta}(t)\right) \geq 0 \quad \forall v \in \mathcal{V},
$$

where

$$
\sigma^{\alpha, \beta}:=M^{\top}\left(B(t)^{\top} \lambda^{\alpha, \beta}(t)+r(t)\right)+\alpha v^{\alpha, \beta}(t)+\beta e,
$$

with $e:=(1, \ldots, 1)^{\top} \in \mathbb{R}^{2 m}$. The adjoint equation (1) as well as the adjoint variables $\lambda^{\alpha, \beta}$ do not change im comparison to Problem $\left(\mathrm{PQ}_{\alpha, \beta}\right)$. A detailed discussion of the optimality conditions can be found in [3] and $[9]$.

\section{$5 \quad$ Uniqueness of solutions}

It is well known that the solution of Problem $\left(\overline{T_{\alpha, \beta}}\right)$ is uniquely determined for each $\beta \geq 0$, if $\alpha>0$ (compare e.g. [13, Satz 3.2.5]). This extends with (5) to Problem $\left(\mathrm{PQ}_{\alpha, \beta}\right)$.

In the case of $\alpha=0$ we consider a fixed parameter $\beta^{*} \geq 0$ and assume that the optimal control $v^{0, \beta^{*}}$ of Problem $\mathrm{TQ}_{0, \beta^{*}}$ is of bang-bang type which implies an optimal control $u^{0, \beta^{*}}$ of bang-zero-bang type for Problem $\mathrm{PQ}_{0, \beta^{*}}$ by (5). To ensure this we assume that

(B1) There exists a solution $\left(x^{0, \beta^{*}}, v^{0, \beta^{*}}\right) \in \mathcal{T}$ of $\left(\mathrm{TQ}_{0, \beta^{*}}\right)$ such that the set $\Sigma$ of zeros of the components of the switching function $\sigma^{0, \beta^{*}}$ defined by (7) is finite and $0, t_{f} \notin \Sigma$, i.e. $\Sigma=\left\{s_{1}, \ldots, s_{l}\right\}$ with $0<s_{1}<\ldots<s_{l}<t_{f}$.

Let $\mathcal{I}\left(s_{j}\right):=\left\{1 \leq i \leq 2 m \mid \sigma_{i}^{0, \beta^{*}}\left(s_{j}\right)=0\right\}$ be the set of active indices for the components of the switching function. In order to get stability of the bang-bang structure under perturbations we need an additional assumption (compare [14]): 
(B2) The functions $B$ and $r$ are differentiable, $\dot{B}$ and $\dot{r}$ are Lipschitz continuous, and there exists $\bar{\sigma}>0$ such that

$$
\min _{1 \leq j \leq l} \min _{i \in \mathcal{I}\left(s_{j}\right)}\left\{\left|\dot{\sigma}_{i}^{0, \beta^{*}}\left(s_{j}\right)\right|\right\} \geq 2 \bar{\sigma} .
$$

Remark 2. Assumption (B2) can be slightly relaxed (see e.g. [15/9]).

The following result is extracted from [14, Proof of Lemma 3.3]. Proofs can also be found in [2], 15] and [9].

Lemma 1. Let $\left(x^{0, \beta^{*}}, v^{0, \beta^{*}}\right)$ be a minimizer for Problem $\left(\mathrm{TQ}_{0, \beta^{*}}\right)$ and let the switching function $\sigma^{0, \beta^{*}}(t)$ be defined by (7). If Assumptions (B1) and (B2) are satisfied, then there are constants $\omega, \gamma, \bar{\delta}>0$ independent of $\beta$ such that for any feasible pair $(x, v)$

$$
\int_{0}^{t_{f}} \sigma^{0, \beta^{*}}(t)^{\top}\left(v(t)-v^{0, \beta^{*}}(t)\right) \mathrm{d} t \geq \omega\left\|v-v^{0, \beta^{*}}\right\|_{L^{1}}^{2}
$$

if $\left\|v-v^{0, \beta^{*}}\right\|_{L^{1}} \leq 2 \gamma \bar{\delta}$, and

$$
\int_{0}^{t_{f}} \sigma^{0, \beta^{*}}(t)^{\top}\left(v(t)-v^{0, \beta^{*}}(t)\right) \mathrm{d} t \geq \omega\left\|v-v^{0, \beta^{*}}\right\|_{L^{1}}
$$

if $\left\|v-v^{0, \beta^{*}}\right\|_{L^{1}} \geq 2 \gamma \bar{\delta}$.

By the help of standard arguments this result implies uniqueness of the solution of $\left(\mathrm{TQ}_{0, \beta^{*}}\right)$ (compare [14, Theorem 2.2]). It follows with (5) that Problem $\left(\mathrm{PQ}_{0, \beta^{*}}\right)$ has a unique solution, too.

\section{Calmness of solutions}

In this section for $\alpha \geq 0$ and $\beta \geq 0$ we denote by $\left(x^{\alpha, \beta}, u^{\alpha, \beta}\right)$ and $\left(x^{\alpha, \beta}, v^{\alpha, \beta}\right)$ the solutions of $\left(\mathrm{PQ}_{\alpha, \beta}\right)$ and $\left(\mathrm{TQ}_{\alpha, \beta}\right)$, respectively. We want to study the dependence of solutions on $\alpha$ and $\beta$. We derive estimates which show that the solutions as functions of the regularization parameters $\alpha$ and $\beta$ are calm at $\alpha=0$ and $\beta=\beta^{*}$ (compare Dontchev/Rockafellar [16, Sect. 1C]). For this purpose we combine the results achieved in [9] and [2].

Theorem 1. Let (B1) and (B2) be satisfied for some $\beta^{*} \geq 0$. Then for any $\alpha \geq 0$ and $\beta \geq 0$ the estimate

$$
\left\|v^{\alpha, \beta}-v^{0, \beta^{*}}\right\|_{L^{1}} \leq c_{1}\left(\alpha+\left|\beta-\beta^{*}\right|\right)
$$

holds, where the constant $c_{1}$ is independent of $\alpha$ and $\beta$. 
Proof. We only consider the case $\left\|v^{\alpha, \beta}-v^{0, \beta^{*}}\right\|_{L^{1}} \leq 2 \gamma \bar{\delta}$ and refer to 9 ] and 2 ] for the case $\left\|v^{\alpha, \beta}-v^{0, \beta^{*}}\right\|_{L^{1}} \geq 2 \gamma \bar{\delta}$ which can be handled analogously. Since Assumptions (B1) and (B2) are satisfied, for $\alpha, \beta \geq 0$ by (8) we have

$$
\int_{0}^{t_{f}} \sigma^{0, \beta^{*}}(t)^{\top}\left(v^{\alpha, \beta}(t)-v^{0, \beta^{*}}(t)\right) \mathrm{d} t \geq \omega\left\|v^{\alpha, \beta}-v^{0, \beta^{*}}\right\|_{L^{1}}^{2}
$$

with $\omega>0$. By the minimum principle (6) we obtain

$$
\int_{0}^{t_{f}} \sigma^{\alpha, \beta}(t)^{\top}\left(v^{0, \beta^{*}}(t)-v^{\alpha, \beta}(t)\right) \mathrm{d} t \geq 0 .
$$

Adding (12) and (11) it follows that

$$
\int_{0}^{t_{f}}\left(\sigma^{0, \beta^{*}}(t)-\sigma^{\alpha, \beta}(t)\right)^{\top}\left(v^{\alpha, \beta}(t)-v^{0, \beta^{*}}(t)\right) \mathrm{d} t \geq \omega\left\|v^{\alpha, \beta}-v^{0, \beta^{*}}\right\|_{L^{1}}^{2} .
$$

Since

$$
\sigma^{0, \beta^{*}}(t)-\sigma^{\alpha, \beta}(t)=\mathcal{B}(t)^{\top}\left(\lambda^{0, \beta^{*}}(t)-\lambda^{\alpha, \beta}(t)\right)+\left(\beta^{*}-\beta\right) e-\alpha v^{\alpha, \beta}(t),
$$

and due to the fact that $x^{\alpha, \beta}, x^{0, \beta^{*}}$ satisfy the system equation, and $\lambda^{\alpha, \beta}, \lambda^{0, \beta^{*}}$ satisfy the adjoint equation we obtain

$$
\begin{aligned}
\int_{0}^{t_{f}} & {\left[\mathcal{B}(t)^{\top}\left(\lambda^{0, \beta^{*}}(t)-\lambda^{\alpha, \beta}(t)\right)\right]^{\top}\left(v^{\alpha, \beta}(t)-v^{0, \beta^{*}}(t)\right) \mathrm{d} t } \\
= & \left(x^{0, \beta^{*}}\left(t_{f}\right)-x^{\alpha, \beta}\left(t_{f}\right)\right)^{\top} Q\left(x^{\alpha, \beta}\left(t_{f}\right)-x^{0, \beta^{*}}\left(t_{f}\right)\right) \\
& +\int_{0}^{t_{f}}\left(x^{0, \beta^{*}}(t)-x^{\alpha, \beta}(t)\right)^{\top} W(t)\left(x^{\alpha, \beta}(t)-x^{0, \beta^{*}}(t)\right) \mathrm{d} t .
\end{aligned}
$$

Together with 13 this implies

$$
\begin{aligned}
& \omega\left\|v^{\alpha, \beta}-v^{0, \beta^{*}}\right\|_{L^{1}}^{2}+\left(x^{\alpha, \beta}\left(t_{f}\right)-x^{0, \beta^{*}}\left(t_{f}\right)\right)^{\top} Q\left(x^{\alpha, \beta}\left(t_{f}\right)-x^{0, \beta^{*}}\left(t_{f}\right)\right) \\
& \quad+\int_{0}^{t_{f}}\left(x^{\alpha, \beta}(t)-x^{0, \beta^{*}}(t)\right)^{\top} W(t)\left(x^{\alpha, \beta}(t)-x^{0, \beta^{*}}(t)\right) \mathrm{d} t \\
& \leq \int_{0}^{t_{f}}\left[\left(\beta^{*}-\beta\right) e-\alpha v^{\alpha, \beta}(t)\right]^{\top}\left(v^{\alpha, \beta}(t)-v^{0, \beta^{*}}(t)\right) \mathrm{d} t \\
& \leq\left|\beta-\beta^{*}\right|\left\|v^{\alpha, \beta}-v^{0, \beta^{*}}\right\|_{L^{1}}+\alpha\left\|v^{\alpha, \beta}\right\|_{L^{\infty}}\left\|v^{\alpha, \beta}-v^{0, \beta^{*}}\right\|_{L^{1}} .
\end{aligned}
$$

Since the matrices $Q$ and $W(t), t \in\left[0, t_{f}\right]$, are assumed to be positive semidefinite and $\omega>0$, we obtain

$$
\omega\left\|v^{\alpha, \beta}-v^{0, \beta^{*}}\right\|_{L^{1}}^{2} \leq\left(\left|\beta-\beta^{*}\right|+\alpha\left\|v^{\alpha, \beta}\right\|_{L^{\infty}}\right)\left\|v^{\alpha, \beta}-v^{0, \beta^{*}}\right\|_{L^{1}} .
$$

We now get 10 with some constant $c_{1}$ independent of $\alpha$ and $\beta$. 
Remark 3. By Theorem 1 we also obtain estimates for the optimal states

$$
\left\|x^{\alpha, \beta}-x^{0, \beta^{*}}\right\|_{1,1} \leq \bar{c}_{1}\left(\alpha+\left|\beta-\beta^{*}\right|\right)
$$

and for the optimal controls $u^{\alpha, \beta}$ of the original problem $\left(\mathrm{PQ}_{\alpha, \beta}\right)$, by using the matrix (4) and the relation (5) between $u^{\alpha, \beta}$ and $v^{\alpha, \beta}$

$$
\begin{aligned}
\left\|u^{\alpha, \beta}-u^{0, \beta^{*}}\right\|_{L^{1}} & =\left\|M v^{\alpha, \beta}-M v^{0, \beta^{*}}\right\|_{L^{1}} \leq\|M\|_{1}\left\|v^{\alpha, \beta}-v^{0, \beta^{*}}\right\|_{L^{1}} \\
& \leq c_{1}\left(\alpha+\left|\beta-\beta^{*}\right|\right) .
\end{aligned}
$$

If we choose some $\beta$ in a sufficiently small neighborhood of $\beta^{*}$ this result can even be improved.

Theorem 2. Let (B1) and (B2) be satisfied for some $\beta^{*} \geq 0$. Then there exist $\rho>0$ and a constant $c_{2}$ independent of $\alpha \geq 0$ and $\rho$, such that for any $\beta_{i} \in \mathbb{R}$, $i=1,2$, with $\beta_{i} \geq 0$ and $\left|\beta_{i}-\beta^{*}\right|<\rho$ the estimate

$$
\left\|v^{\alpha, \beta_{1}}-v^{0, \beta_{2}}\right\|_{L^{1}} \leq c_{2}\left(\alpha+\left|\beta_{1}-\beta_{2}\right|\right)
$$

holds.

Proof. We use [9, Theorem 6.3, Remark 10], which proved the local Lipschitzcontinuity of the optimal control depending on $\beta$, where the constant $\tilde{c}$ is independent of $\beta$ :

$$
\left\|u^{0, \beta_{1}}-u^{0, \beta_{2}}\right\|_{L^{1}} \leq \tilde{c}\left|\beta_{1}-\beta_{2}\right| .
$$

In addition to this we are able to extend the result of [2, Theorem 4.1] using the problem transformation introduced in Sect. 4 and obtain

$$
\left\|u^{\alpha, \beta_{1}}-u^{0, \beta_{1}}\right\|_{L^{1}} \leq \bar{c} \alpha
$$

with some constant $\bar{c}$ independent of $\alpha$. Together 15 and 16 lead to

$$
\begin{aligned}
\left\|u^{\alpha, \beta_{1}}-u^{0, \beta_{2}}\right\|_{L^{1}} & \leq\left\|u^{\alpha, \beta_{1}}-u^{0, \beta_{1}}\right\|_{L^{1}}+\left\|u^{0, \beta_{1}}-u^{0, \beta_{2}}\right\|_{L^{1}} \\
& \leq \tilde{c} \alpha+\bar{c}\left|\beta_{1}-\beta_{2}\right|,
\end{aligned}
$$

which implies 14 .

\section{Discretization}

For the numerical solution of Problem $\left(\mathrm{PQ}_{\alpha, \beta}\right)$ we use the Euler discretization scheme described in [15] and [9]. Given a natural number $N$ and let $h_{N}=t_{f} / N$ be the meshsize, we approximate the cost functional $f_{\alpha, \beta}$ by

$$
\begin{aligned}
f_{\alpha, \beta, N}(x, u)= & \frac{1}{2} x_{N}^{\top} Q x_{N}+q^{\top} x_{N}+h_{N} \sum_{i=0}^{N-1} \frac{1}{2} x_{i}^{\top} W\left(t_{i}\right) x_{i}+w\left(t_{i}\right)^{\top} x_{i}+r\left(t_{i}\right)^{\top} u_{i} \\
& +h_{N}\left(\beta \sum_{i=0}^{N-1} \sum_{j=1}^{m}\left|u_{j, i}\right|+\frac{\alpha}{2} \sum_{i=0}^{N-1} \sum_{j=1}^{m} u_{j, i}^{2}\right),
\end{aligned}
$$


and Problem $\left(\overline{\left.\mathrm{PQ}_{\alpha, \beta}\right)}\right.$ by

$$
\begin{aligned}
& \min f_{\alpha, \beta, N}(x, u) \\
& \text { s.t. } x_{i+1}=x_{i}+h_{N}\left(A\left(t_{i}\right) x_{i}+B\left(t_{i}\right) u_{i}\right), i=0, \ldots, N-1, \quad\left(\mathrm{PQ}_{\alpha, \beta}^{\mathrm{N}}\right) \\
& x_{0}=a \text {, } \\
& u_{i} \in U, \quad i=0, \ldots, N-1 .
\end{aligned}
$$

Remark 4. Note that analogously to 9 we solve a transformed discretized problem (compare also Sect. 4) to compute the solution of Problem $\left(\overline{\mathrm{PQ}_{\alpha, \beta}}\right)$ numerically.

Theorem 3. Let $\left(x^{0, \beta^{*}}, u^{0, \beta^{*}}\right)$ be the solution of Problem $\sqrt{\mathrm{PQ}_{0, \beta^{*}}}$ for which Assumptions (B1) and (B2) are satisfied. Then, for sufficiently large $N$, choosing $\alpha=c_{\alpha} h_{N}$ and $\beta=\beta^{*}+c_{\beta} h_{N}$ with constants $c_{\alpha}$ and $c_{\beta}$, any optimal control

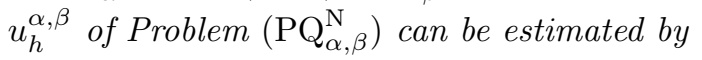

$$
\left\|u_{h}^{\alpha, \beta}-u^{0, \beta^{*}}\right\|_{L^{1}} \leq c_{u} h_{N},
$$

where the constant $c_{u}$ is independent of $N$.

Proof. Using [17, Theorem 5.2] and [9, Theorem 5.1, Remark 8] we have

$$
\begin{aligned}
\left\|u_{h}^{\alpha, \beta}-u^{0, \beta^{*}}\right\|_{L^{1}} & \leq\left\|u_{h}^{\alpha, \beta}-u^{0, \beta}\right\|_{L^{1}}+\left\|u^{0, \beta}-u^{0, \beta^{*}}\right\|_{L^{1}} \\
& \leq c_{\alpha} h+\tilde{c}_{\beta}\left|\beta-\beta^{*}\right|
\end{aligned}
$$

with some constant $\tilde{c}_{\beta}$ independent of $\beta$, which implies the assertion.

Example 1 (The Rocket Car). We consider the popular example of the rocket car, driving from some starting point to it's destination $(0,0)$.

$$
\begin{array}{ll}
\min & \frac{1}{2}\left(x_{1}(5)^{2}+x_{2}(5)^{2}\right)+\beta\|u\|_{L^{1}}+\frac{\alpha}{2}\|u\|_{L^{2}}^{2} \\
\text { s.t. } & \dot{x}_{1}(t)=x_{2}(t), \quad \dot{x}_{2}(t)=u(t) \text { a.e. on }[0,5], \\
& x_{1}(0)=6, \quad x_{2}(0)=1, \\
& u(t) \in[-1,1]
\end{array}
$$

Table 1 1shows numerical results for different meshsizes which confirm the theoretical findings of Theorem 3 . To solve the discretized problems we used Ipopt 18.

Table 1. Discretization for different $N, \beta^{*}=1, \beta=\beta^{*}+h_{N}$ and $\alpha=10 h_{N}$.

\begin{tabular}{ccccccc}
\hline$N$ & 125 & 250 & 500 & 1000 & 2000 & 4000 \\
\hline$\left\|u_{h}^{\alpha, \beta}-u^{0, \beta^{*}}\right\|_{L^{1}}$ & 0.2644 & 0.1344 & 0.0644 & 0.0331 & 0.0177 & 0.0083 \\
$\frac{\left\|u_{h}^{\alpha, \beta}-u^{0, \beta^{*}}\right\|_{L^{1}}}{h_{N}}$ & 6.6098 & 6.7177 & 6.4409 & 6.6123 & 7.0826 & 6.6752 \\
\hline
\end{tabular}




\section{References}

1. Deckelnick, K., Hinze, M.: A note on the approximation of elliptic control problems with bang-bang controls. Computational Optimization and Applications 51(2) (2012) 931-939

2. Alt, W., Seydenschwanz, M.: Regularization and discretization of linear-quadratic control problems. Control and Cybernetics 40(4) (2011) 903-920

3. Vossen, G., Maurer, H.: On $L^{1}$-minimization in optimal control and applications to robotics. Optimal Control Applications and Methods 27(6) (2006) 301-321

4. Sakawa, Y.: Trajectory planning of a free-flying robot using the optimal control. Optimal Control Applications and Methods 20(5) (1999) 235-248

5. Stadler, G.: Elliptic optimal control problems with $L^{1}$-control cost and applications for the placement of control devices. Computational Optimization and Applications 44(2) (2009) 159-181

6. Casas, E., Herzog, R., Wachsmuth, G.: Approximation of sparse controls in semilinear equations by piecewise linear functions. Numerische Mathematik 122(4) (2012) 645-669

7. Casas, E., Herzog, R., Wachsmuth, G.: Optimality conditions and error analysis of semilinear elliptic control problems with $L^{1}$-cost functional. SIAM Journal on Optimization 22(3) (2012) 795-820

8. Wachsmuth, G., Wachsmuth, D.: Convergence and regularization results for optimal control problems with sparsity functional. ESAIM: Control, Optimisation and Calculus of Variations 17(3) (2011) 858-886

9. Alt, W., Schneider, C.: Linear-Quadratic Control Problems with $L^{1}$-Control Cost. Optimal Control Applications and Methods (2014) to appear.

10. Ekeland, I., Temam, R.: Convex Analysis and Variational Problems. Volume 1 of Studies in Mathematics and its Applications. North-Holland Publishing Company (1976)

11. Clarke, F.H.: Functional Analysis, Calculus of Variations and Optimal Control. Springer (2013)

12. Murty, K.G.: Operations Research: Deterministic Optimization Models. PrenticeHall (1995)

13. Alt, W., Schneider, C., Seydenschwanz, M.: EAGLE-STARTHILFE Optimale Steuerung. Theorie und Verfahren. Edition am Gutenbergplatz Leipzig (2013)

14. Felgenhauer, U.: On Stability of Bang-Bang Type Controls. SIAM Journal on Control and Optimization 41(6) (2003) 1843-1867

15. Alt, W., Baier, R., Gerdts, M., Lempio, F.: Error Bounds for Euler Approximation of Linear-Quadratic Control Problems with Bang-Bang Solutions. Numerical Algebra, Control and Optimization 2(3) (2012) 547-570

16. Dontchev, A.L., Rockafellar, R.T.: Implicit Functions and Solution Mappings. Springer (2009)

17. Seydenschwanz, M.: Improved Error Estimates for Discrete Regularization of Linear-Quadratic Control Problems with Bang-Bang Solutions. submitted (2013)

18. Wächter, A., Biegler, L.T.: On the implementation of an interior-point filter linesearch algorithm for large-scale nonlinear programming. Mathematical Programming 106(1) (2006) 25-57 